\title{
Niyobyum İlavesinin (Bi, Pb)-2223 Bulk Süperiletken Sistemine Etkisi Üzerine Bir Araştırma
}

\author{
An Investigation on the Influence of Niobium Adding on (Bi,Pb)-2223 Bulk Superconducting \\ System
}

Hasan A $\breve{G I L} *, 1$

${ }^{1}$ Hakkari Üniversitesi, Mühendislik Fakültesi, Malzeme Bilimi ve Mühendisliği Bölümü, 30000, Hakkari, Türkiye

• Geliş tarihi / Received: 08.06.2020 • • Düzeltilerek geliş tarihi / Received in revised form: 18.09 .2020 • Kabul tarihi / Accepted: 30.09 .2020

\section{$\ddot{O} z$}

Niyobyum $(\mathrm{Nb})$ ilaveli $\mathrm{Bi}_{1.85} \mathrm{~Pb}_{0.35} \mathrm{Sr}_{1.9} \mathrm{Ca}_{2.1} \mathrm{Cu}_{3.1} \mathrm{O}_{\delta}$ içeren bir dizi bizmut-tabanlı bulk süperiletken malzemeler, geleneksel katı hal reaksiyon yöntemi ile sentezlendi. Malzemelerin fazları ve mikro-yapısal analizi, X-1şını kırınımı (XRD) ve taramalı elektron mikroskobu (SEM) ile gerçekleştirildi. Niyobyum ilavesinin elektriksel ve manyetik özellikler üzerindeki etkisi, sırasıyla özdirenç ve mıknatıslanma ölçümleri ile belirlendi. Niyobyum ilavesiyle kritik sıcaklıkta önemli bir azalma olmadı. Ayrıca niyobyum ilave edilmiş malzemelerin oda sıcaklığı özdirenci, saf malzemeye kıyasla azaldı. Nb katkılı numunelerin kritik akım yoğunluk değerleri saf numuneninkinden daha düşüktür.

Anahtar kelimeler: Akı Çivilemesi, (Bi, Pb)-2223 Süperiletkeni, Kritik Akım Yoğunluğu, $\mathrm{Nb}_{2} \mathrm{O}_{5} \mathrm{Katk}_{1 \mathrm{~S}}$

\begin{abstract}
A series of bismuth-based bulk superconducting materials with niobium-added $\mathrm{Bi}_{1.85} \mathrm{~Pb}_{0.35} \mathrm{Sr}_{1.9} \mathrm{Ca}_{2.1} \mathrm{Cu}_{3.1} \mathrm{O}_{\delta}$ were synthesized by the conventional solid state reaction method. The phases and microstructural analysis of the samples were carried out by X-ray diffraction (XRD) and scanning electron microscopy (SEM). The effect of niobium addition on electrical and magnetic properties was determined by resistivity and magnetization measurements, respectively. There was no significant reduction in critical temperature by the addition of niobium. Also the room temperature resistivity of the niobium added samples decreased compared to the pristine sample. The critical current density values of the samples with $\mathrm{Nb}$ addition were lower than the pure sample.
\end{abstract}

Keywords: Flux Pinning, (Bi, Pb)-2223 Superconductor, Critical Current Density, $\mathrm{Nb}_{2} \mathrm{O}_{5}$ Addition

*a Hasan AĞIL; hasanagil@ hakkari.edu.tr, Tel: (0438) 21212 12, orcid.org/0000-0002-1574-3232 


\section{Giriş}

$\mathrm{Bi}-\mathrm{Sr}-\mathrm{Ca}-\mathrm{Cu}-\mathrm{O}$ süperiletken ailesi (BSCCO), umut vaat eden teknik uygulamalar açısından birçok araştırma başlatmıştır. Özellikle Maeda tarafindan keşfedilen Bi-2223 fazı, yüksek kritik sıcaklığı nedeniyle özelliklerinin iyileştirilmesi için birçok araştırmacı tarafından hala araştırılmaktadır (Maeda vd., 1988). Sistemdeki kimyasal yer değiştirmeler veya kimyasal katkılama çalışmaları, Bi-tabanlı bileşiklerde faz yapısının kökenini anlamak, yeni fazları sentezlemek ve süperiletkenlik mekanizmasını anlamak için önemlidir. Bi-2223 fazının üretimi sırasında diğer fazlar (Bi-2201 ve Bi-2212) da oluşabilir. Ǘç fazın tümü nedeniyle BSCCO, sistemin tek bir faz olarak oluşmasını önleyen benzer oluşum enerjilerine sahiptir (Grivel vd., 1996). Bi bölgesine kısmen $\mathrm{Pb}$ eklenerek Bi-2223 fazının kararlılığında önemli bir iyileşme gözlenmiştir (Grivel vd., 1996). Bi-2223 bulk malzemeler düşük kritik akım yoğunluğuna $\left(J_{c}\right)$ sahip olmalarına rağmen, diğer yüksek sıcaklık süperiletkenlerine kıyasla iyi mekanik özellikleri ve kolay şekillendirilebilirliği ile en yüksek kritik sicaklığa $\left(T_{c}\right)$ sahiptirler (Rouessac vd., 1997). Bi tabanlı süperiletken malzemelerin uygulamalarındaki temel sinırlamalar, tanecik sınırlarındaki zayıf bağlar ve akı çivileme kabiliyetleridir (Cursino vd., 2009). Başlangıç stokiyometrisi, başlangıç tozlarının saflığı ve boyutu, toz hazırlama koşulu, presleme basıncı, 1sıl işlem süreci ve katkı maddelerinin türü ve miktarı, parçacık bağlılığı ve ak1 çivileme yeteneğini önemli ölçüde etkiler. BSCCO'nun pratik uygulamalarda kullanımı için yüksek kritik akım yoğunluğu gerektiğinden, bu süperiletkenin süperiletken özelliklerini iyileştiren etkili yapay çivileme merkezlerinin oluşturulması önemlidir (Albiss vd., 2010; Zouaoui vd., 2008; Schneemeyer vd., 1987). Bu bağlamda, BSCCO süperiletkeninin kritik parametrelerini (kritik sıcaklık, kritik akım yoğunluğu, kritik manyetik alan, vb.) iyileştirmek için çok çaba sarf edilmiştir. Son zamanlarda, $\mathrm{K}, \mathrm{Eu}, \mathrm{Tm}_{2} \mathrm{O}_{3}, \mathrm{In}_{2} \mathrm{O}_{3}$, $\mathrm{Ga}_{2} \mathrm{O}_{3}, \mathrm{NiO}, \mathrm{Ni}_{0.5} \mathrm{Zn}_{0.5} \mathrm{Fe}_{2} \mathrm{O}_{4}, \mathrm{WO}_{3}, \mathrm{TiO}_{2}$ gibi nano boyutlu elementlerin, bileşiklerin ve oksitlerin etkisi, BSCCO'nun süperiletken özelliklerini iyileştirmek için kapsamlı bir şekilde araştırılmıştır (Tran vd., 2019; Padam vd., 2019; Ulgen vd., 2018; de Vera vd., 2019; Guner vd., 2019; Harabor vd., 2019; Mohd Suib vd., 2019; Rahal vd., 2019; Ulgen vd., 2019). Nano parçacıkların tanecik-içi ve tanecik-arası bölgelerde etkin çivileme merkezleri olarak davrandıkları bulunmuştur (Ishii vd., 2000).
Bi-tabanlı yüksek sıcaklık süperiletkenleri için genel formül $\mathrm{Bi}_{2} \mathrm{Sr}_{2} \mathrm{Ca}_{n-1} \mathrm{Cu}_{n} \mathrm{O}_{2 n+4}$ olarak ifade edilebilir. Burada $\mathrm{n}$, c eksenine dik farkl $\mathrm{Cu}-\mathrm{O}$ düzlemleri için $(\mathrm{n}=1,2,3)$ değerlerini alabilir. Fazlar genellikle, Bi-2201, Bi-2212, Bi-2223 olarak $\mathrm{Bi}: \mathrm{Sr}: \mathrm{Ca}: \mathrm{Cu}$ bileşen elementlerinin atomik oranıyla, $\mathrm{CuO}_{2}$ tabakalarının sayısına atıfta bulunarak $\mathrm{n}=1,2,3$ olarak belirtilir ve sirasiyla 20, 95 ve 110 K'lik süperiletken geçiş sıcaklıklarına sahip fazlardır (Gul vd., 2005).

Literatürde ayrıca Bi-tabanlı süperiletkenlere niyobyum ilavesinin veya katkısının etkileri ile ilgili çalışmalarda bulunmaktadır. Ancak bu çalışmalar ile bizim çalışmamız arasında hem malzeme hazırlama hem başlangıç bileşimi hem de gerçekleştirilen karakterizasyonlar arasında bazı farklılıklar bulunmaktadır. Ghazanfari vd. tarafından $\mathrm{Nb}$ ilavesinin BSCCO'nun süperiletken özelliklerine etkileri araştırıldı (Ghazanfari vd., 2007). Bu çalışmada tüm başlangıç tozları birlikte karıştırılmış ve ardından tozlar kalsine edilerek malzemeler pelet haline getirilmiştir. $\mathrm{Bu}$ çalışmanın, mevcut çalışmadan farkı malzeme hazırlama aşamasıdır. Bilgili vd. yaptığı çalışmada ise hem başlangıç bileşimi hem karıştırma işlemi hem de 1 sıl işlem süreçleri açısından bizim çalışmamız ile arasında farklılıklar bulunmaktadır (Bilgili vd., 2014). Asghari vd. yaptığı başka bir çalışmada ise kurşundan $(\mathrm{Pb})$ eksiltme yapilarak yerine niyobyum $(\mathrm{Nb})$ katkısı yapılarak malzeme üretimi gerçekleştirilmiştir (Asghari vd., 2017).

$\mathrm{Bu}$ çalışmanın amacı, Nb ilavesinin Bi-2223 bulk malzemelerin çivileme mekanizması ve faz oluşumu üzerindeki etkisini araştırmaktır. Sonuç olarak, niyobyum ilavesinin faz hacim oranını arttırmak için Bi-2223 bulk süperiletkenleri üzerindeki olası rolü elektriksel, manyetik ve yapısal ölçümlerle araştırıldı.

\section{Materyal ve Metod}

Geleneksel kat1 hal reaksiyon yöntemi, $\mathrm{Bi}_{1.85} \mathrm{~Pb}_{0.35} \mathrm{Sr}_{1.9} \mathrm{Ca}_{2.1} \mathrm{Cu}_{3.1} \mathrm{O}_{\delta} /\left(\mathrm{Nb}_{2} \mathrm{O}_{5}\right)_{\mathrm{x}} \quad$ bulk süperiletkenini üretmek için kullanıldı; burada $\mathrm{x}$ ağırlıkça $0,0,0,1,0,2,0,5,0,7$ ve 1,0 'dır. $\mathrm{Bu}$ malzemeler bundan sonra sirasiyla A, B, C, D, E ve $\mathrm{F}$ olarak adlandırılacaktır. Yüksek saflıkta başlangıç tozları $\left(\mathrm{Bi}_{2} \mathrm{O}_{3}(\%\right.$ 99,999), $\mathrm{PbO}(\%$ 99,999), $\mathrm{SrCO}_{3}$ (\% 99,995), $\mathrm{CaCO}_{3}(\%$ 99,999) ve $\mathrm{CuO}(\% 99,99))$ stokiyometrik orana göre hassas bir şekilde tartıldı ve bir agat havan içerisinde tokmak ile karıştırıldı ve öğütüldü. Toz karışımı daha sonra 12 saat boyunca hava ortaminda sirasiyla $800{ }^{\circ} \mathrm{C}$ ve $820{ }^{\circ} \mathrm{C}$ de bir kül firında 
kalsine edildi. Bu karışım, kalsinasyon işlemleri sırasında agat havan ve tokmak ile ara öğütmeye tabi tutuldu. $\mathrm{Bu}$ işlemden sonra nihai toz karışımına ağırlıkça (toplam toz karışımının) \% 0 , $0,1,0,2,0,5,0,7$ ve 1 oranında niyobyum ilave edildi ve daha sonra $1 \mathrm{~mm}$ kalınlığında ve $13 \mathrm{~mm}$ çapında malzemeler $375 \mathrm{MPa}$ basınç altında preslendi. Daha sonra tüm numuneler bir tüp firın içerisinde hava ortamında $835{ }^{\circ} \mathrm{C}^{\prime}$ de 40 saat sinterlendi. Nihai malzemeler, Bi-2223 faz hacim oranını, $T_{c}$ ve $J_{c}$ 'yi arttırmak için, yeniden ögütüldü ve $650 \mathrm{MPa}$ basınç altında yeniden preslendi (Safran vd., 2017). Son olarak sinterleme işlemi bir tüp firın içerisinde $835^{\circ} \mathrm{C}$ 'de 40 saat gerçekleştirildi.

Tüm örneklerin yapısal analizi ve faz tespiti $\mathrm{X}$ ışını toz kırınım yöntemi (XRD) ile araştırıldı. Örgü parametreleri, X-1şını verilerindeki kırınım pikleri kullanılarak UnitCell programı ile hesaplandı. Örneklerin morfolojisini ve tanecik büyüklüğünü incelemek için tarama elektron mikroskobu (SEM) kullanıldı. Elektriksel ve manyetik özellikler sırasıyla doğru akım (d.a.) direnç ve mıknatıslanma ölçümleri ile belirlendi. $\mathrm{Bu}$ ölçümler Quantum Design PPMS (fiziksel özellikler ölçüm sistemi) ile alındı. Kritik akım yoğunluğu Bean formülü ile hesaplandı (Cagliero vd., 2007).

\section{Bulgular ve Tartışma}

$\mathrm{Bi}_{1.85} \mathrm{~Pb}_{0.35} \mathrm{Sr}_{1.9} \mathrm{Ca}_{2.1} \mathrm{Cu}_{3.1} \mathrm{O}_{\delta} /\left(\mathrm{Nb}_{2} \mathrm{O}_{5}\right)_{\mathrm{x}} \quad$ bulk süperiletkenlerin XRD desenleri Şekil 1'de gösterilmektedir. (hkl) Miller indisleri MDI Jade 6 programı kullanılarak belirlendi ve XRD piklerinde işaretlendi. Tüm örneklerin XRD desenlerinde tüm piklerin Bi-2223 fazına ait olduğu ve yabancı faz bulunmadığı gözlendi. Bu, malzeme üretim sürecinin başariyla gerçekleştirildiğini gösterir. İndekslenmiş piklerin (hkl) değerinden hesaplanan örgü parametrelerinde, sistematik bir değişiklik yoktu. $\mathrm{Bu}$ değerler Tablo 1'de gösterildi

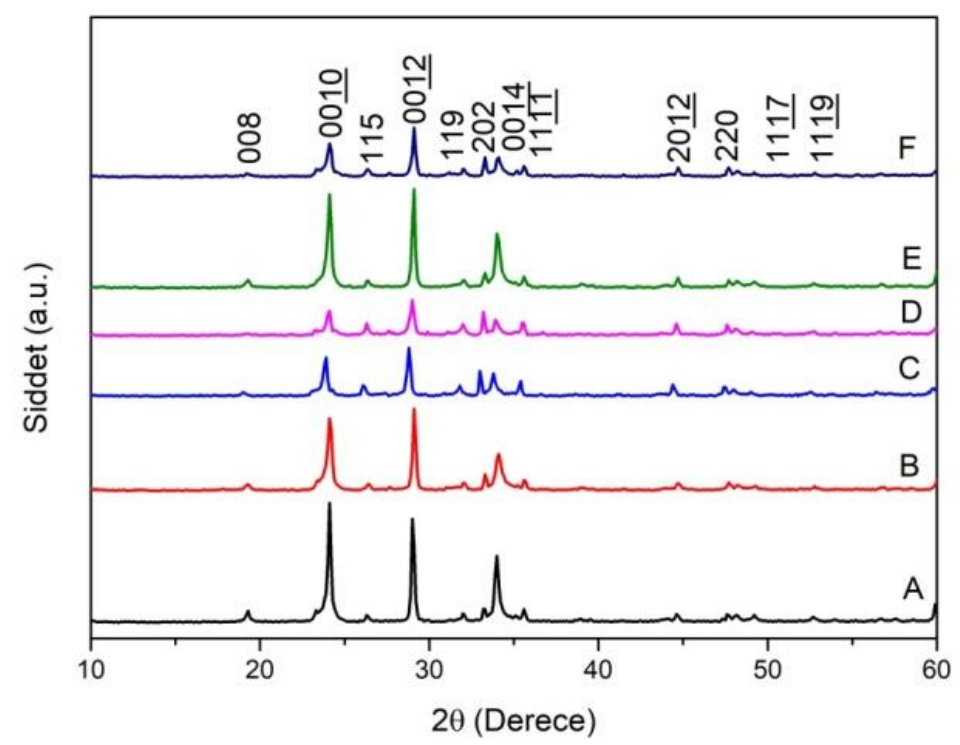

Şekil 1. (Bi, Pb)-2223 + ağ. \% x Nb $\mathrm{Nb}_{5}$ bulk süperiletkenlerin XRD desenleri.

Tanecik yapısı, yüksek sıcaklık süperiletkenlerin en önemli özelliklerinden birisidir (Silver vd., 2002). Örneklerin mikro yapısı, yüzey morfolojisi ve tanecik yapısı SEM görüntüleri ile incelendi. İkincil elektron (SE) görüntüleme ve 10000 büyütme kullanıldı Şekil 2, $\mathrm{Bi}_{1.85} \mathrm{~Pb}_{0.35} \mathrm{Sr}_{1.9} \mathrm{Ca}_{2.1} \mathrm{Cu}_{3.1} \mathrm{O}_{\delta} /\left(\mathrm{Nb}_{2} \mathrm{O}_{5}\right)_{\mathrm{x}} \quad$ bulk süperiletkenlerin SEM görüntülerini göstermektedir. Plaka benzeri bir parçacık morfolojisi, $(\mathrm{Bi}, \mathrm{Pb})-2223$ sisteminde $\operatorname{artan} \mathrm{Nb}$ ilavesiyle artar. Görüntülerde görüldüğü gibi yüzey morfolojisi, (Bi, $\mathrm{Pb}$ )-2223 sisteminde artan $\mathrm{Nb}$ ilavesiyle değişir. Plaka benzeri tanecik morfolojisi, ağırlıkça \% 0,5 Nb ilavesinden sonra gözlendi. Öte yandan, katkı1ı malzemelerin tanecik büyüklügünün ve plaka kalınlığının, artan $\mathrm{Nb}$ ilavesi miktarıyla azaldığı gözlenmektedir. Artan $\mathrm{Nb}$ miktarı ile tanecikler keskin kenarlara ve köşelere sahip olmaya ve daha ince bir yapıya kavuşmaya başladı. Parçacıklar mikro yapıda rastgele dağ $11 d$. 

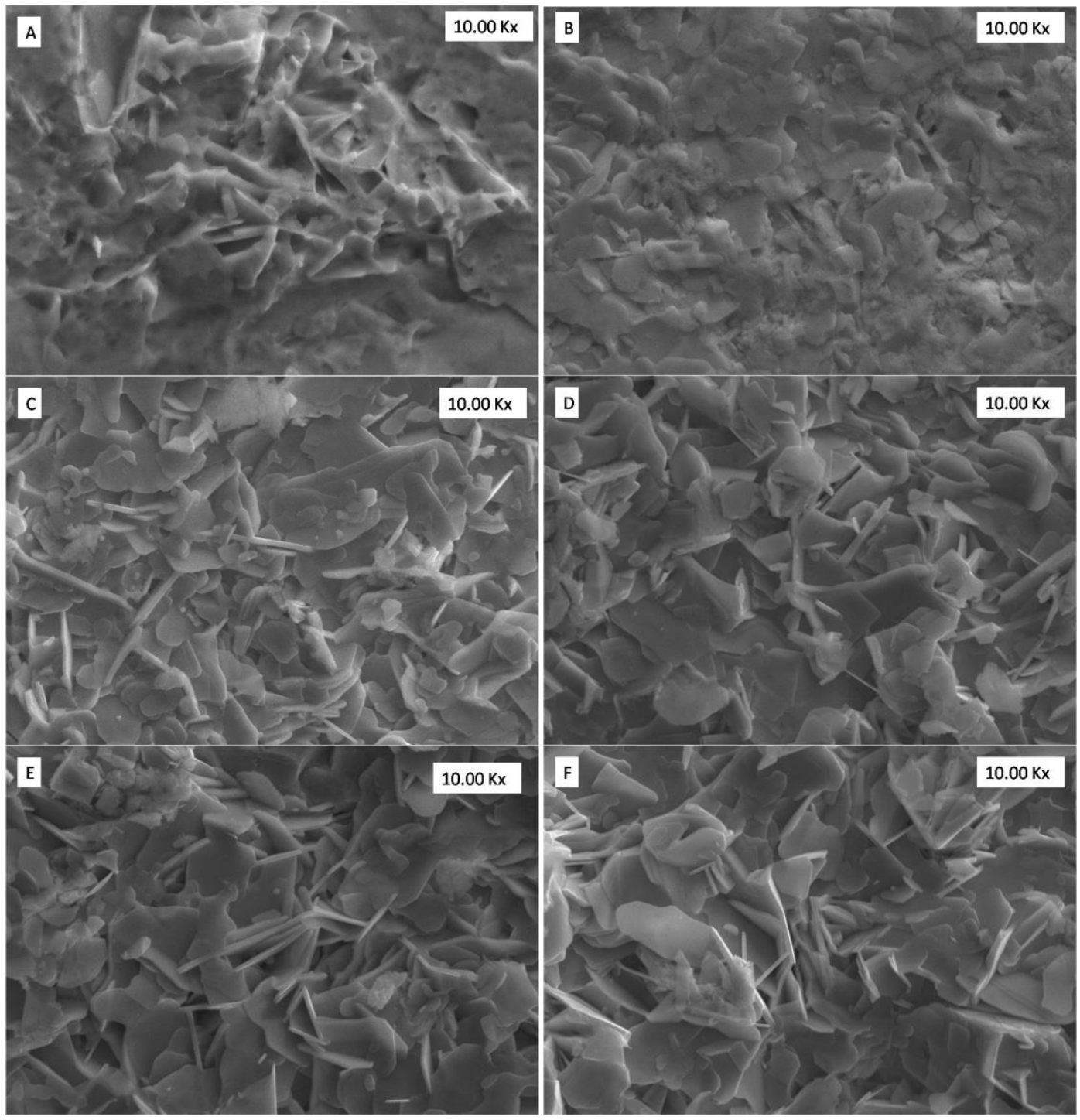

Şekil 2. Tüm malzemelerin SEM görüntüleri.

Niyobyum ilavelerinin, numunelerin geçiş sıcaklıkları üzerindeki etkilerini anlamak için $\mathrm{T}=$ 10-300 K aralığında elektriksel özdirenç ölçümleri yapıld1. $\quad \mathrm{Bi}_{1.85} \mathrm{~Pb}_{0.35} \mathrm{Sr}_{1.9} \mathrm{Ca}_{2.1} \mathrm{Cu}_{3.1} \mathrm{O}_{\delta} /\left(\mathrm{Nb}_{2} \mathrm{O}_{5}\right)_{\mathrm{x}}$ bileşikleri için elektrik özdirencinin $(\rho(\mathrm{T}))$ sıcaklık bağımlılı̆̆ı Şekil 3 'te gösterilmektedir. Tüm numuneler geçiş sıcaklı̆̆ının üzerinde metalik davranış gösterir. Saf $(\mathrm{Bi}, \mathrm{Pb})-2223$ ile karşılaştırıldığında, oda sıcaklığ 1 özdirencinin $\left(\rho_{0}\right)$ niyobyum miktarındaki artışla azaldığ 1 açıktır. Numunelerin $\mathrm{T}_{\mathrm{c} \text {,başlangı̣ }}$ (normal durum direncinden ilk sapma ile tanımlanır (Yamamoto vd., 2015)) değerleri neredeyse değişmezken, niyobyum miktarındaki artışla $T_{\mathrm{c}, \text { stfir }}$ (sıfır direnç kritik sıcaklığı ile tanımlanır (Yamamoto vd., 2015)) değerleri azalır. $\mathrm{Bu}$ nedenle, geçiş genişliği $\left(\Delta \mathrm{T}_{\mathrm{c}}\right)$ de niyobyum miktarındaki artışla azald1 (Yamamoto vd., 2015). Tablo 2'de tüm malzemeler için bu parametreler verildi.

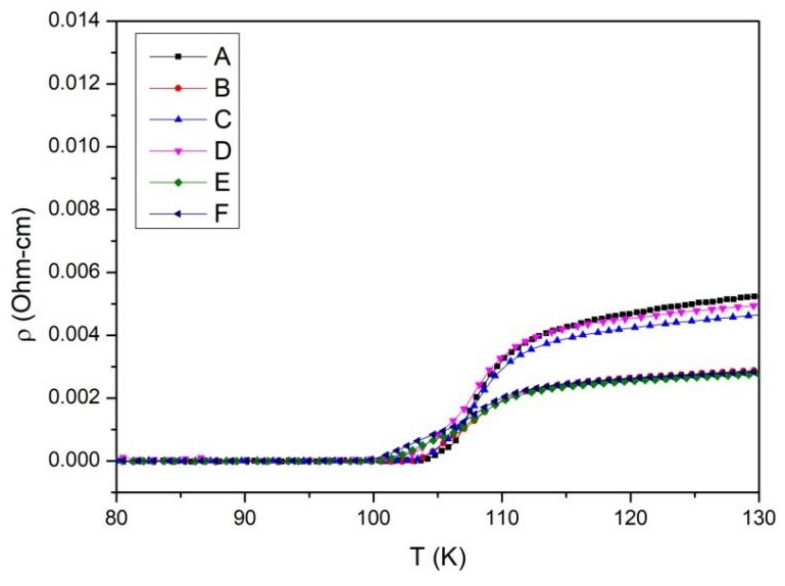

Şekil 3. $\mathrm{Bi}_{1.85} \mathrm{~Pb}_{0.35} \mathrm{Sr}_{1.9} \mathrm{Ca}_{2.1} \mathrm{Cu}_{3.1} \mathrm{O}_{\delta} /\left(\mathrm{Nb}_{2} \mathrm{O}_{5}\right)_{\mathrm{x}}$ bileşikleri için sıcaklığa karşı elektriksel özdirenç. 
Tablo 1. Nb katkılı (Bi, Pb)-2223 bileşikleri için örgü parametreleri.

\begin{tabular}{|l|l|l|}
\hline Ă̆ırlıkça \% x & $\mathbf{a}(\mathbf{\AA})$ & $\mathbf{c}(\mathbf{\AA})$ \\
\hline 0 & 5.41084 & 36.69625 \\
\hline 0.1 & 5.39966 & 36.63906 \\
\hline 0.2 & 5.43361 & 36.86053 \\
\hline 0.5 & 5.41112 & 36.71241 \\
\hline 0.7 & 5.39825 & 36.70500 \\
\hline 1 & 5.39944 & 36.66236 \\
\hline
\end{tabular}

$\mathrm{Bi}_{1.85} \mathrm{~Pb}_{0.35} \mathrm{Sr}_{1.9} \mathrm{Ca}_{2.1} \mathrm{Cu}_{3.1} \mathrm{O}_{\delta} /\left(\mathrm{Nb}_{2} \mathrm{O}_{5}\right)_{\mathrm{x}}$ bileşiklerinin mıknatıslanmasının manyetik alan bağımlılığı Şekil 4'te gösterilmektedir. Miknatıslanma ölçümleri $(\mathrm{M}-\mathrm{H}) \mathrm{T}=10 \mathrm{~K}$ ve $\mathrm{H}= \pm 8$ Tesla'da gerçekleştirildi. Niyobyum miktarındaki artışla, eğrilerin genişliğinin azaldığı açıtır. Histerezis eğrisinin genişliğinin azalması kristal boyutu ve yüzeyde kusur birikimi ile açıklanabilir (Kishio vd., 1991).

Tablo 2. Nb katkılı (Bi, Pb)-2223 bileşikleri için kritik parametreleri.

\begin{tabular}{|c|c|c|c|c|c|c|}
\hline Ăğırlıkça \%x & $T_{c, \text { basslanglç }}(K)$ & $T_{c, s, f i r}(K)$ & $\Delta T_{c}(K)$ & $\rho_{0}(O h m-\mathrm{cm})$ & $B(T)$ & $J_{c}\left(A / c^{2}\right)$ \\
\hline 0 & 111.36 & 104.83 & 6.53 & 0.01123 & $\begin{array}{l}8 \\
0\end{array}$ & $\begin{array}{l}6.5 \times 10^{3} \\
3.2 \times 10^{4}\end{array}$ \\
\hline 0.1 & 110.89 & 103.08 & 7.81 & 0.00867 & $\begin{array}{l}8 \\
0\end{array}$ & $\begin{array}{l}4.2 \times 10^{3} \\
2.1 \times 10^{4}\end{array}$ \\
\hline 0.2 & 110.51 & 100.26 & 10.25 & 0.00944 & $\begin{array}{l}8 \\
0\end{array}$ & $\begin{array}{l}2.9 \times 10^{3} \\
1.3 \times 10^{4}\end{array}$ \\
\hline 0.5 & 110 & 100.26 & 9.74 & 0.00533 & $\begin{array}{l}8 \\
0 \\
\end{array}$ & $\begin{array}{l}5.7 \times 10^{3} \\
3.1 \times 10^{4}\end{array}$ \\
\hline 0.7 & 110 & 100.26 & 9.74 & 0.0051 & $\begin{array}{l}8 \\
0 \\
\end{array}$ & $\begin{array}{l}5.3 \times 10^{3} \\
2.5 \times 10^{4} \\
\end{array}$ \\
\hline 1 & 110.86 & 103.00 & 7.86 & 0.00435 & $\begin{array}{l}8 \\
0\end{array}$ & $\begin{array}{l}3.4 \times 10^{3} \\
1.8 \times 10^{4}\end{array}$ \\
\hline
\end{tabular}

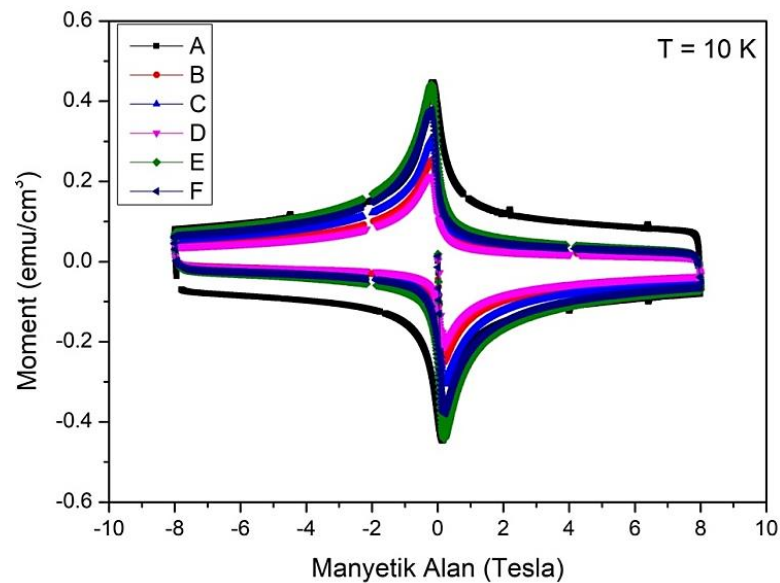

Şekil 4. $\mathrm{Bi}_{1.85} \mathrm{~Pb}_{0.35} \mathrm{Sr}_{1.9} \mathrm{Ca}_{2.1} \mathrm{Cu}_{3.1} \mathrm{O}_{\delta} /\left(\mathrm{Nb}_{2} \mathrm{O}_{5}\right)_{\mathrm{x}}$ bileşikleri için $\mathrm{M}(\mathrm{H})$ histerezis eğrileri.

$\mathrm{J}_{\mathrm{c}}$ değerleri Bean modeli kullanılarak $\mathrm{M}(\mathrm{H})$ eğrilerinin genişliğinden aşağıdaki eşitlikle hesaplandı (Bean, 1962):

$$
J_{c}=\frac{20 \Delta M}{a\left(1-\frac{a}{3 b}\right)}
$$

burada $\Delta \mathrm{M}$ manyetik histerezis döngülerinin genişliği, $a$ ve $b$ ise numunenin kesit boyutlarıdır $(\mathrm{a}<\mathrm{b})$ Şekil 5, $\mathrm{Bi}_{1.85} \mathrm{~Pb}_{0.35} \mathrm{Sr}_{1.9} \mathrm{Ca}_{2.1} \mathrm{Cu}_{3.1} \mathrm{O}_{\delta} /\left(\mathrm{Nb}_{2} \mathrm{O}_{5}\right)_{\mathrm{x}} \quad$ bileşikleri için $\mathrm{J}_{\mathrm{c}}$ 'nin uygulanan manyetik alan bağımlılığını gösterir. Niyobyum ilavesi ile manyetik alana bağımlılığın azaldığı açıktır. Saf (Bi, Pb)-2223 numunesinin, niyobyum katk1lı numunelerden daha yüksek $J_{c}$ değerlerine sahip olduğu gözlendi. Bunun sebebinin malzemeye eklenen safsızlıklar ile uygulanan manyetik alanın numuneye nüfuz etmeye başlaması ve dolayısıyla kritik akım yoğunluğunun azalmasına yol açtı̆̆ düşünülmektedir (Bilgili vd., 2017). Bu sonuçlar ak1 çivileme özelliklerinin niyobyum ilavesiyle bozulduğunu göstermektedir (Bilgili vd., 2017).

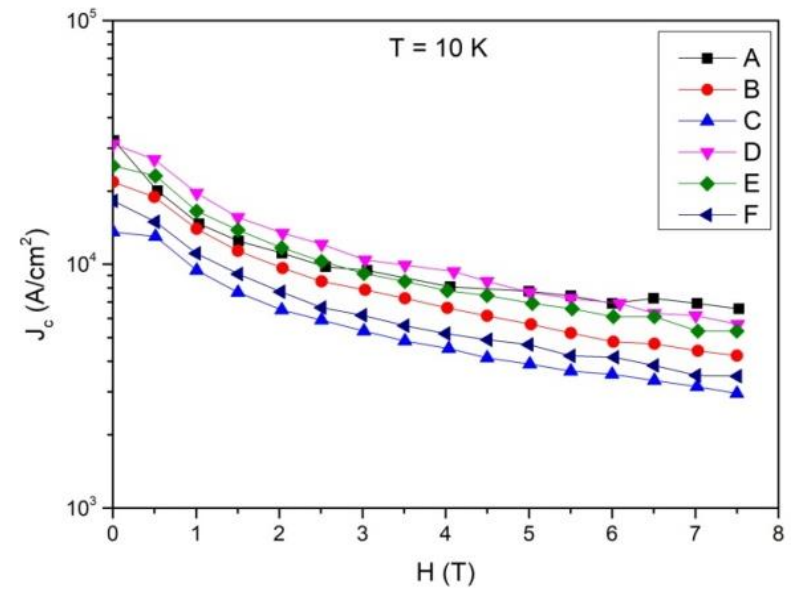

Şekil 5. $\mathrm{T}=10 \quad \mathrm{~K}$ 'da $\mathrm{Bi}_{1.85} \mathrm{~Pb}_{0.35} \mathrm{Sr}_{1.9} \mathrm{Ca}_{2.1} \mathrm{Cu}_{3.1} \mathrm{O}_{\delta} /\left(\mathrm{Nb}_{2} \mathrm{O}_{5}\right)_{\mathrm{x}} \quad$ bileşikleri için uygulanan manyetik alana karşı $\mathrm{J}_{\mathrm{c}}$ değişimleri 


\section{Tartışma ve Sonuçlar}

$\mathrm{Bu}$ çalışmada, niyobyum ilavesinin (Bi, Pb)-2223 bileşiklerinin yapısal, morfolojik, elektriksel ve manyetik özellikleri üzerine etkileri araştırıldı. XRD analizi ağılıklı olarak tek fazlı tetragonal $(\mathrm{Bi}, \quad \mathrm{Pb})-2223$ fazını gösterir. Yüzey morfolojilerinin niyobyum ilavesiyle değiştiği gözlendi. Süperiletken geçiş sicaklıklarının niyobyum ilavesiyle değişmediği, ancak geçiş genişliklerinin arttığı bulundu. Katkı miktarı arttıkça normal durum direnci azaldı. $M(H)$ sonuçlarının analizi, kritik akım yoğunluğu değerlerinin azaldığını gösterir. $\mathrm{Bu}$, numunelerin akım taşıma kapasitesinin, az miktarda niyobyum ilavesiyle bozulduğu anlamına gelir.

\section{Teşekkür}

$\mathrm{Bu}$ çalışma Hakkari Üniversitesi Bilimsel Araştırma Projeleri Birimi tarafindan FM19AYP1 nolu proje kapsamında desteklenmiştir.

\section{Kaynaklar}

Albiss, B.A., Obaidat, I.M., Gharaibehb, M., Ghamlouche, H. ve Obeidat, S.M., 2010. Impact of Addition of Magnetic Nanoparticles on Vortex Pinning and Microstructure Properties of $\mathrm{Bi}-\mathrm{Sr}-\mathrm{Ca}-\mathrm{Cu}-\mathrm{O}$ Superconductor. Solid State Communications, 150, 1542-1547.

Asghari, R., Arslan, L.C., Sedghi, H. ve Naghshara, H., 2017. Synthesis and Characterization of $\mathrm{Nb}$ Substitution on (Bi-Pb)-2223 Superconductors. Journal of Low Temperature Physics, 189, 1526.

Bean, C.P., 1962. Magnetization of Hard Superconductors. Physical Review Letters, 8, 250.

Bilgili, O. ve Kocabaş, K., 2014. Effect of Nb Addition on Magnetic, Structural and Superconducting Properties of (Bi, $\mathrm{Pb})-2223$ Superconductors. Journal of Materials Science: Materials in Electronics, 25, 2889-2897.

Cagliero, S., Agostino, A., Bonometti, E. ve Truccato, M., 2007. Electrical Transport Effects due to Oxygen Content Modifications in a $\mathrm{Bi}_{2} \mathrm{Sr}_{2} \mathrm{CaCu}_{2} \mathrm{O}_{8+\delta} \quad$ Superconducting Whisker. Superconductor Science and Technology, 20, 667.

Cursino, E., Schmool, D.S., Garitaonandia, J.S. ve Rodrigues, D., 2009. Artificial Pinning Center Studies in Bi2212 Tapes and Bulks With Zirconium Oxide Inclusion. IEEE Transactions on Applied Superconductivity, 19(3), 35163519. de Vera, F.I., Bardolaza, H., Arcilla, C. ve Sarmago, R., 2019. Effect of $\mathrm{In}_{2} \mathrm{O}_{3}$ on the Grain Connectivity and Superconducting Behavior of $\mathrm{Bi}_{2} \mathrm{Sr}_{2-x} \mathrm{In}_{x} \mathrm{CaCu}_{2} \mathrm{O}_{8+\mathrm{d}}$. SN Applied Sciences, 1, 96.

Ghazanfari, N., Kılıç, A., Gencer, A. ve Özkan, H., 2007. Effects of $\mathrm{Nb}_{2} \mathrm{O}_{5}$ Addition on Superconducting Properties of BSCCO. Solid State Communications, 144, 210-214.

Grivel, J.C. ve Flukiger, R., 1996. Factors Influencing the Apparent Activation Energy for the Formation of the $(\mathrm{Bi}, \mathrm{Pb})_{2} \mathrm{Sr}_{2} \mathrm{Ca}_{2} \mathrm{Cu}_{3} \mathrm{O}_{10+\delta}$ Compound. Journal of Alloys and Compounds, 241, 127-133.

Gul, I.H., Rehman, M.A., Ali, M. ve Maqsood, A., 2005. Effect of Vanadium and Barium on the Bi-based (2223) Superconductors. Physica C: Superconductivity and its Applications, 432, 7180.

Guner, S.B., Zalaoglu, Y., Turgay, T., Ozyurt, O., Ulgen, A.T., Dogruer, M. ve Yildirim, G., 2019. A Detailed Research for Determination of $\mathrm{Bi} / \mathrm{Ga}$ Partial Substitution Effect in Bi-2212 Superconducting Matrix on Crucial Characteristic Features. Journal of Alloys and Compounds, 772, 388-398.

Harabor, A., Rotaru, P. ve Harabor, N.A., 2019. Effect of Ni Substitute in Off-Stoichiometric $\mathrm{Bi}(\mathrm{Pb})$ $\mathrm{Sr}-\mathrm{Ca}-\mathrm{Cu}(\mathrm{Ni})-\mathrm{O}$ Superconductor. Excess Conductivity, XRD Analysis and Thermal Behaviour. Ceramics International, 45, 27422750 .

Ishii, A. ve Hatano, T., 2000. Preparation of High Quality $\mathrm{Bi}_{2} \mathrm{Sr}_{2} \mathrm{CaCu}_{2} \mathrm{O}_{8+\delta}$ Thin Films on a $\mathrm{MgO}$ Substrate by Pulsed Laser Ablation and PostAnnealing - Recrystallization of Films Accompanying In-Plane Rotation of $a$ and $b$ Axes. Physica C, 340, 173-177.

Kishio, K., Komiya, S., Motohira, N., Kitazawa, K. ve Yamafuji, K., 1991. A Novel Method to Avoid Demagnetization Effect in Magnetization Studies of $\mathrm{Bi}_{2} \mathrm{Sr}_{2} \mathrm{CaCu}_{2} \mathrm{O}_{8}$ Single Crystals with the Field Perpendicular to the $\mathrm{CuO}_{2}$ Plane. Physica C, 185, 2377-2378.

Maeda, H., Tanaka, Y., Fukutomi, M. ve Asano, T., 1988. A New High- $T_{\mathrm{c}}$ Oxide Superconductor without a Rare Earth Element. Japanese Journal of Applied Physics, 27(2), L209-L210.

Mohd Suib, N.R., Abu Bakar, I.P., Ibrahim, N.B. ve Abd-Shukor, R., 2019. Critical Current Density and $\mathrm{AC}$ Susceptibility of $\mathrm{Bi}_{1.6} \mathrm{~Pb}_{0.4} \mathrm{Sr}_{2} \mathrm{Ca}_{2} \mathrm{Cu}_{3} \mathrm{O}_{10}$ Superconductor with $\quad \mathrm{Ni}_{0.5} \mathrm{Zn}_{0.5} \mathrm{Fe}_{2} \mathrm{O}_{4}$ Nanomaterial. Journal of Superconductivity and Novel Magnetism, 32, 145-150. 
Padam, G.K., Arora, M., Kaushik, S.D. ve Ekbote, S.N., 2019. Enhanced Critical Current Density $\left(J_{\mathrm{c}}\right)$ and Fractural Strength of Low and High Eu Level Doped Bare Bulk (Bi, Pb)-2223 Rods for Cryogenic Applications. Physica C: Superconductivity and its applications, 562, 7884.

Rahal, H.T., Awad, R., Abdel-Gaber, A.M., Marhaba, S. ve Abou-Aly, A.I., 2019. A Comparative Study on the Influence of the Addition of Different Nano-Oxide Particles on the Thermopower of (Bi,Pb)-2223 Superconductor. Applied Physics A, 125, 365.

Rouessac, V., Desgardin, G. ve Gomina, M., 1997. Influence of the Sinter-Forging Conditions on the Mechanical Properties of Textured Bulk (Bi,Pb)-2223 Ceramics. Physica C, 282-287, 2573-2574.

Safran, S., Kilıc, A. ve Ozturk, O., 2017. Effect of RePelletization on Structural, Mechanical and Superconducting Properties of BSCCO Superconductors. Journal of Materials Science: Materials in Electronics, 28, 1799-1803.

Schneemeyer, L.F., Waszczak, J.V., Siegrist, T., van Dover, R.B. ve Rupp, L.W., 1987. Superconductivity in $\mathrm{YBa}_{2} \mathrm{Cu}_{3} \mathrm{O}_{7}$ Single Crystals. Nature, 328, 601-603.

Silver, T., Pan, A.V., Ionescu, M., Qin, M.J. ve Dou, S.X., 2002. Developments in High Temperature Superconductivity. Annual Reports Section "C" (Physical Chemistry), 98, 323-373.

Tran, D.H., Pham, A.T., Le, T.M., Anh, D.T.K., Phan, Y.T., Man, N.K., Pham, D. ve Kang, W.N., 2019. Enhanced Flux Pinning Properties in $\mathrm{Bi}_{1.6} \mathrm{~Pb}_{0.4} \mathrm{Sr}_{2-\mathrm{x}} \mathrm{K}_{\mathrm{x}} \mathrm{Ca}_{2} \mathrm{Cu}_{3} \mathrm{O}_{10+\delta} \quad$ Compounds. Journal of Materials Science: Materials in Electronics, 30, 8233-8238.

Ulgen, A.T., Turgay, T., Terzioglu, C., Yildirim, G. ve $\mathrm{Oz}, \mathrm{M} ., 2018$. Role of $\mathrm{Bi} / \mathrm{Tm}$ Substitution in Bi2212 System on Crystal Structure Quality, Pair Wave Function and Polaronic States. Journal of Alloys and Compounds, 764, 755-766.

Ulgen, A.T. ve Yildirim, G., 2019. Degradation in Fundamental Characteristic Features of Bi-2212 Superconducting Ceramic Material with $\mathrm{Sr} / \mathrm{Ti}$ Partial Substitution. Journal of Materials Science: Materials in Electronics, 30, 82688277.

Yamamoto, A., Takeshita, N., Terakura, C. ve Tokura, Y., 2015. High Pressure Effects Revisited for the Cuprate Superconductor Family with Highest Critical Temperature. Nature Communications, 6, 8990.
Zouaoui, M., Ghattas, A., Annabi, M., Ben Azzouz, F. ve Ben Salem, M., 2008. Effect of Nano-Size $\mathrm{ZrO}_{2}$ Addition on the Flux Pinning Properties of (Bi, $\mathrm{Pb}$ )-2223 Superconductor. Superconductor Science and Technology, 21, 125005. 